\title{
Cetuximab retreatment in patients with metastatic colorectal cancer who exhibited a clinical benefit in response to prior cetuximab: A retrospective study
}

\author{
HIROAKI TANIOKA $^{1,2}$, MOTOI ASANO ${ }^{1}$, RYOUSUKE YOSHIDA ${ }^{3}$, NAOHISA WAKI $^{3}$, FUTOSHI UNO $^{3}$, \\ MASAHIRO ISHIZAKI ${ }^{3}$, KAZUKI YAMASHITA ${ }^{3}$, YUKI MORISHITA ${ }^{4}$ and TAKESHI NAGASAKA ${ }^{2}$ \\ ${ }^{1}$ Department of Medical Oncology, Okayama Rosai Hospital, Okayama 702-8055; \\ ${ }^{2}$ Department of Clinical Oncology, Kawasaki Medical School Hospital, Okayama 701-0192; \\ Departments of ${ }^{3}$ Surgery and ${ }^{4}$ Pharmacy, Okayama Rosai Hospital, Okayama 702-8055, Japan
}

Received November 14,2017; Accepted April 5, 2018

DOI: $10.3892 / \mathrm{ol} .2018 .9127$

\begin{abstract}
Clinical benefits of cetuximab retreatment in patients with metastatic colorectal (mCRC) have been reported. In the present study, the effect of cetuximab retreatment on predictive markers was investigated by evaluating the clinical benefit of initial cetuximab treatment prior to cetuximab retreatment. Between November 2012 and March 2017, 14 patients with KRAS proto-oncogene GTPase exon 2 wild-type mCRC who exhibited a clinical benefit (confirmed stable disease for at least 6 months or a clinical response) to an initial cetuximab-based regimen, who received multiple lines of chemotherapy following disease progression and ultimately received a second cetuximab and irinotecan regimen, were retrospectively analyzed. For retreatment, patients received bi-weekly irinotecan $\left(120-150 \mathrm{mg} / \mathrm{m}^{2}\right)$ combined with cetuximab $\left(400 \mathrm{mg} / \mathrm{m}^{2}\right.$ as an initial dose, followed by $250 \mathrm{mg} / \mathrm{m}^{2}$, weekly). The median age of the 14 patients (11 males, 3 females) was 68 years (32-77). The median progression-free survival (PFS) following prior cetuximab-based therapy was 6.6 months (range, 4.1-18.4). Initial cetuximab treatment was administered as a first-line treatment in 11 patients, a second-line treatment in 1 patient and a third-line treatment in 2 patients. The median interval
\end{abstract}

Correspondence to: Dr Hiroaki Tanioka, Department of Medical Oncology, Okayama Rosai Hospital, 1-10-25 Chikkou-Midorimachi, Okayama 702-8055, Japan

E-mail: hiroakit130@gmail.com

Abbreviations: mCRC, metastatic colorectal cancer; ORR, objective response rate; PFS, progression-free survival; CRC, colorectal cancer; EGFR, epidermal growth factor receptor; RECIST, Response Evaluation Criteria in Solid Tumors; CTCAE, Common Terminology Criteria for Adverse Events; DCR, disease control rate; CECT, contrast-enhanced computed tomography

Key words: cetuximab, colorectal cancer, retreatment, re-challenge, irinotecan time between the last cycle of initial cetuximab-based therapy and the first cycle of cetuximab retreatment was 13.1 months (range, 6.0-37.1). The objective response rate of cetuximab retreatment was $21.4 \%$ and the median PFS was 4.4 months (95\% confidence interval, 1.4-5.6). The Spearman's correlation coefficient for the PFS following retreatment and duration of initial cetuximab-based regimens demonstrated a more marked correlation compared with that between the PFS following retreatment and the interval time between the two regimens $(\mathrm{r}=0.45, \mathrm{P}=0.11$ vs. $\mathrm{r}=0.08, \mathrm{P}=0.79)$. Cetuximab retreatment may provide clinical benefit to patients with mCRC who were good responders with longer periods of initial cetuximab-based therapy.

\section{Introduction}

Colorectal cancer (CRC) is the second-most commonly diagnosed cancer and the third leading cause of cancer mortality in Japan (1). A total of $20 \%$ of patients with CRC present with metastatic disease at the time of diagnosis, and, in addition, $17 \%$ of patients who undergo curative resection will experience a relapse of CRC (2). Standard chemotherapy for metastatic CRC (mCRC) based on cytotoxic agents (fluoropyrimidines, irinotecan, and oxaliplatin) and monoclonal antibodies, including those targeting vascular endothelial growth factor (bevacizumab) and those targeting epidermal growth factor receptor (EGFR) (cetuximab and panitumumab), has demonstrated well-established clinical activity (2-4). Particularly, cetuximab and panitumumab as standard combination chemotherapy regimens in first-line treatment have led to a marked improvement in clinical outcomes for patients with Ras wild-type $\operatorname{mCRC}(5,6)$. Previously, trifluridine/tipiracil (TAS-102) and regorafenib monotherapies have demonstrated improvement in overall survival in patients with CRC disease progression following standard therapies, and these drugs remain additional treatment options $(7,8)$. However, in addition to the improvement in survival for patients with $\mathrm{mCRC}$, a number of patients who exhibited good disease control following chemotherapy also exhibited good long-term performance status and remain 
suitable for additional therapy following progression beyond third- or fourth-line treatment (9). At present, a standard treatment for those who are resistant towards or unable to tolerate these agents does not exist.

Previously, a small number of studies have suggested the clinical benefit of a retreatment strategy with anti-EGFR-based therapy in patients with mCRC who exhibited a clinical benefit following prior administration of anti-EGFR antibodies (10-12). In a phase II prospective study, Santini et al (11) investigated retreatment with cetuximab plus irinotecan in 39 patients with KRAS proto-oncogene GTPase (KRAS) wild-type mCRC who exhibited disease progression following an initial clinical response to first-line cetuximab-based therapy. The median time to progression with prior cetuximab treatment was 10 months; the median interval time between the last cycle of initial cetuximab-based therapy and first cycle of cetuximab retreatment was 6 months. Outcomes indicated an objective response rate (ORR) of $53.8 \%$, with 19 partial responses (PR) (48.7\%) and 2 complete responses (CR) (5.1\%), and the median progression-free survival (PFS) was 6.6 months (11).

A retrospective study by Liu et al (13) investigated the efficacy of an anti-EGFR-containing regimen in phase I/II clinical trials in patients with $K R A S$ wild-type mCRC exhibiting disease progression following previous cetuximab or panitumumab therapy. Patients who responded to prior cetuximab or panitumumab therapy were more likely to obtain clinical benefit from retreatment compared with the non-responders. Additionally, prior responders with longer interval lengths between prior anti-EGFR therapy and anti-EGFR retreatment were more likely to respond to anti-EGFR retreatment compared with a shorter interval.

In the present retrospective study, patients with KRAS wild-type unresectable $\mathrm{mCRC}$ who exhibited a clinical benefit from prior cetuximab therapy were selected and received cetuximab retreatment at Okayama Rosai Hospital. The effect of cetuximab retreatment on predictive markers was investigated by evaluating the clinical benefit of initial cetuximab treatment prior to cetuximab retreatment. The results of the present study suggest that cetuximab retreatment provided clinical benefits to patients with $\mathrm{mCRC}$ who exhibited a positive response to longer periods of initial cetuximab-based therapy.

\section{Patients and methods}

Patient selection. Patients with KRAS exon2 wild-type mCRC who were retreated with cetuximab plus irinotecan at Okayama Rosai Hospital (Okayama, Japan) between November 2012 and March 2017 were retrospectively analyzed. All patients exhibited disease progression following an initial clinical benefit [confirmed stable disease (SD) for at least 6 months, complete response (CR) or partial response (PR)] from first-line cetuximab-based therapy and had received multiple cycles of chemotherapy without anti-EGFR antibodies following completion of the initial cetuximab-based therapy.

Cetuximab retreatment. First-line cetuximab combined with FOLFIRI [irinotecan at a dose of $150 \mathrm{mg} / \mathrm{m}^{2}$; leucovorin at a dose of $200 \mathrm{mg} / \mathrm{m}^{2}$; and an intravenous bolus of fluorouracil
(FU) at a dose of $400 \mathrm{mg} / \mathrm{m}^{2}$ followed by a continuous $46 \mathrm{~h}$ infusion of FU at a dose of $2,400 \mathrm{mg} / \mathrm{m}^{2}$ every 2 weeks.)], oxaliplatin-based therapy [FOLFOX (oxaliplatin at a dose of $85 \mathrm{mg} / \mathrm{m}^{2}$; leucovorin at a dose of $200 \mathrm{mg} / \mathrm{m}^{2}$; and an intravenous bolus of FU at a dose of $400 \mathrm{mg} / \mathrm{m}^{2}$ followed by a continuous $46 \mathrm{~h}$ infusion of $\mathrm{FU}$ at a dose of 2,400 mg/m² every 2 weeks.) or SOX (oxaliplatin at a dose of $130 \mathrm{mg} / \mathrm{m}^{2}$ on day 1 and oral S-l at a dose of $80 \mathrm{mg} / \mathrm{m}^{2}$ on days $1-15$ every 3 weeks.)] and irinotecan monotherapy. Some patients received TAS-102 (35 mg/m² given orally twice a day in a 28-day cycle [2-week cycle of 5 days of treatment followed by a 2-day rest period, and then a 14-day rest period]) and regorafenib (160 mg given orally once a day for weeks 1-3 of each 4-week cycle) monotherapies for the interim chemotherapies between cetuximab treatments,

For the retreatment regimen, all patients received irinotecan (150 mg/m ${ }^{2}$ bi-weekly) combined with cetuximab $\left(400 \mathrm{mg} / \mathrm{m}^{2}\right.$ as an initial dose, followed by $250 \mathrm{mg} / \mathrm{m}^{2}$ weekly) every 2 weeks. The dose of irinotecan was modified to $120-150 \mathrm{mg} / \mathrm{m}^{2}$ for each patient based on hematologic or non-hematologic toxicities according to the Common Terminology Criteria for Adverse Events (CTCAE) version 4.0 (14). This treatment was administered until disease progression, unacceptable toxicity, or the physician's decision to terminate treatment. Radiological assessments of tumors were performed by investigators approximately every 8 weeks, and the Response Evaluation Criteria in Solid Tumors (RECIST), version 1.1, was used to assess tumor responses (15). The ORR and the disease control rate (DCR) were defined as the percentage of patients with $\mathrm{CR}$ and PR/CR, $\mathrm{PR}$ and SD respectively. Various toxicities such as rash and hepatological toxities were evaluated every week the patients visited the hospital and were graded according to the CTCAE version 4.0 (14). The Institutional Review Board of Okayama Rosai Hospital approved the present study. Written informed consent was obtained from all patients who participated.

Statistical analysis. One of the objectives of the present study was to assess the correlation between prior cetuximab-based therapy and the effectiveness of retreatment with cetuximab. Spearman's correlation coefficient was utilized for this evaluation. PFS was calculated using the Kaplan-Meier method. All statistical analyses were performed with EZR (Saitama Medical Centre, Jichi Medical University, Saitama, Japan), which is a graphical user interface for R (The R Foundation for Statistical Computing, version 3.2.2; http://www.jichi. ac.jp/saitama-sct/SaitamaHP.files/download.html) (16).

\section{Results}

Patient characteristics. A total of 14 patients with KRAS exon 2 wild-type $\mathrm{mCRC}$ who were retreated with cetuximab plus irinotecan at Okayama Rosai Hospital between November 2012 and March 2017 were analyzed. The median age of the patients was 68 years (range, 32-77). A total of 12 patients exhibited an Eastern Cooperative Oncology Group Performance Status (ECOG-PS) score of 0 to 1, while the remaining patients exhibited a ECOG-PS score of 2 to 3 . Among the 14 patients with KRAS exon 2 (codon 12/13), 12 patients exhibited all-Ras wild-type (KRAS and NRAS proto-oncogene, GTPase). Table I indicates the characteristics 
Table I. Patient characteristics.

\begin{tabular}{lc}
\hline Parameters & $\mathrm{n}$ \\
\hline Sex & \\
Male & 11 \\
Female & 3 \\
Age, median (range) & 68 (32-77) \\
All ras genotype (KRAS/NRAS) & \\
Wild-type & 12 \\
Mutant (KRAS/NRAS) & 2 \\
Eastern Cooperative Oncology & \\
Group Performance Status at baseline & \\
0-1 & 12 \\
2-3 & 2 \\
Organs with metastases & \\
0-2 & 10 \\
3 & 4 \\
Metastatic sites & \\
Lung & \\
Liver & 4 \\
Lymph nodes & 9 \\
Peritoneum & 9 \\
\hline
\end{tabular}

ECOG, Eastern Cooperative Oncology Group; PS performance status.

of all analyzed patients. The initial chemotherapy cycles were as follows: Cetuximab combined with FOLFIRI ( $n=2)$; oxaliplatin-based therapy (FOLFOX or SOX) $(n=10)$; and irinotecan monotherapy $(n=2)$. All patients received cetuximab. Initial cetuximab treatment was administered as a first-line treatment in 11 patients, a second-line treatment in 1 patient (who received FOLFIRI+bevacizumab as first-line therapy) and a third-line treatment in 2 patients (who received FOLFOX+bevacizumab as first-line and FOLFIRI+bevacizumab as second-line therapy). The responses following initial cetuximab-based therapy were 12 cases of PR and 2 cases of SD lasting at least 6 months. All patients exhibited disease progression following a preliminary clinical benefit from initial cetuximab treatment, and no patient defaulted prior treatment due to adverse effects. The median duration of initial cetuximab treatment was 6.6 months (range, 4.1-18.4; Table II).

Outcomes of retreatment. The outcomes of cetuximab retreatment are summarized in Table III. The median interval time between the last cycle of initial cetuximab-based therapy and the first cycle of cetuximab retreatment was 12.6 months (range, 6.6-37.1). The therapeutic lines of cetuximab retreatment, including the initial cetuximab-containing regimens, were as follows: Third-line treatment in 6 patients; fourth-line treatment in 1 patient; fifth-line treatment in 2 patients; and sixth-line treatment in 5 patients. The interim chemotherapies between cetuximab treatments were as follows. Bevacizumab-contained regimens were received in 13 patients,
Table II. Characteristics of initial cetuximab-based therapy.

Treatment characteristics n

$\mathrm{n}$

Therapeutic line of initial cetuximab

1 11

2

1

3

Adjunct chemotherapy regimen FOLFIRI 2 mFOLFOX6/SOX 10 CPT-11 2

Response

CR 0

PR 12

SD $\quad 2$

PD 0

Median duration of cetuximab $\quad 6.6(4.1-18.4)$ treatment, months (range)

FOLFIRI, folinic acid, fluorouracil and irinotecan; FOLFOX, folinic acid, fluorouracil and oxaliplatin; SOX, S-1 and oxaliplatin; CPT-11, irinotecan; CR, Complete response; PR, Partial response; SD, Stable disease; $P D$, progressive disease.

Table III. Characteristics of cetuximab retreatment.

Treatment characteristics $\quad n$

Therapeutic line of cetuximab retreatment

4

5

6

5

Adjunct chemotherapy regimen

CPT-11

Median interval time between prior and cetuximab retreatment, months (range)

$13.1(6.0-37.1)$

CPT-11, irinotecan.

TAS-102 in 7 patients and regorafenib in 6 patients. A total of 7 patients who were retreated with fifth- and sixth-line therapies received TAS-102 $\left(35 \mathrm{mg} / \mathrm{m}^{2}\right.$ given orally twice a day in a 28-day cycle [2-week cycle of 5 days of treatment followed by a 2-day rest period, and then a 14-day rest period]) and regorafenib (160 mg given orally once a day for weeks 1-3 of each 4-week cycle) between the initial and second cetuximab-based therapies. Three patients received FOLFIRI immediately prior to the retreatment. Tumor responses are summarized in Table III. The ORR was $21.4 \%$ (3/14), with CR in 0 patients and PR in 3 patients; the DCR was $71 \%$ (10/14), with CR in 0 patients, $\mathrm{PR}$ in 3 patients, and SD in 7 patients. The median PFS was 4.4 months (95\% confidence interval, 1.4-5.6; Fig. 1). A total of 3 patients who exhibited PR during retreatment were 
Table IV. Adverse events (Common Terminology Criteria for Adverse Events version 4.0).

\begin{tabular}{lcc}
\hline & \multicolumn{2}{c}{ No. of patients (\%) } \\
\cline { 2 - 3 } Adverse events & All grades & $\geq$ Grade 3 \\
\hline Leukopenia & $4(29)$ & $0(0)$ \\
Neutropenia & $5(36)$ & $0(0)$ \\
Hypomagnesemia & $6(43)$ & $0(0)$ \\
Rash & $14(100)$ & $1(7)$ \\
Infusion reactions & $0(0)$ & $0(0)$ \\
Stomatitis & $7(50)$ & $0(0)$ \\
Diarrhea & $5(36)$ & $1(7)$ \\
\hline
\end{tabular}

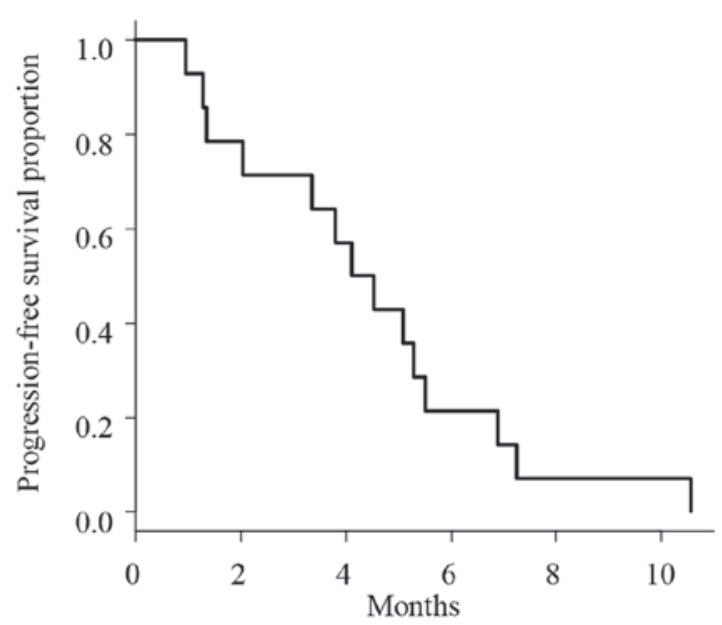

Figure 1. Progression-free survival curve estimated by patients treated with cetuximab retreatment $(n=14)$. The median survival time was 4.4 months.

all-Ras wild-type, with a PS of 0 and had only 1 metastasis site (liver in 2 patients and lymph nodes in 1 patient). The Spearman's correlation coefficient analysis for the PFS of cetuximab retreatment and duration of initial cetuximab treatment $(\mathrm{r}=0.45, \mathrm{P}=0.11)$ demonstrated a more marked correlation compared with that between the PFS of cetuximab retreatment and interval time between initial therapy and retreatment $(r=0.08, \mathrm{P}=0.79$; Fig. 2$)$.

Toxicity of retreatment. Safety assessments were completed based upon all the patients included in the present study. Adverse events of cetuximab retreatment are summarized in Table IV. The most common adverse event was rash $(100 \%)$, followed by stomatitis $(50 \%)$. Grade 3 or 4 toxicities included rash $(7 \%, \mathrm{n}=1)$ and diarrhea $(7 \%, \mathrm{n}=1)$. The majority of adverse events were of grade 1 or 2 toxicity and were manageable. No patients experienced adverse events leading to discontinuation of treatment and there was no infusion reaction with cetuximab.

Case presentation of a patient with a positive response. In this section, we describe a patient who has a PR with cetuximab retreatment. This patient was selected for this case presentation as their liver metastatic tumors exhibited

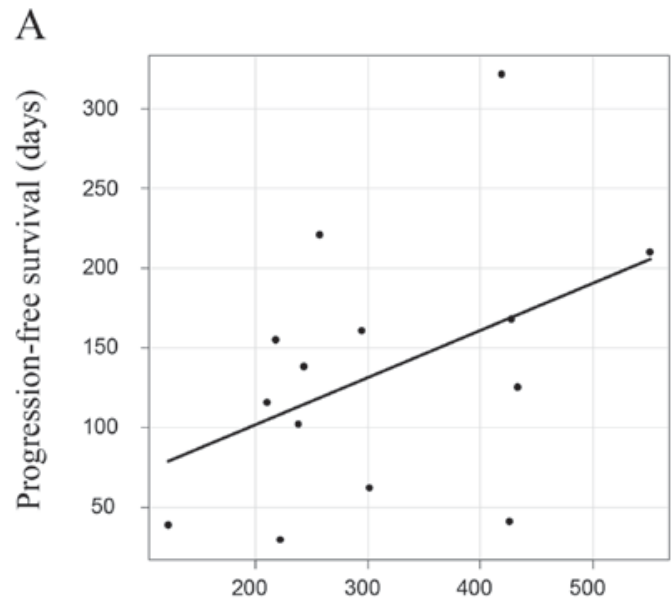

Duration of initial cetuximab treatment (days)

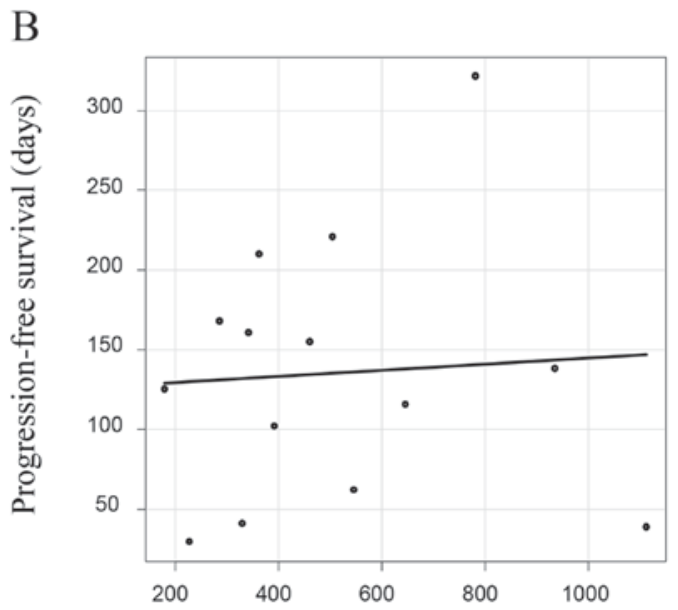

Interval time between prior therapy and retreatment (days)

Figure 2. Spearman's correlation coefficient of the progression-free survival of cetuximab retreatment by (A) duration of initial cetuximab treatment (days) $(\mathrm{r}=0.45, \mathrm{P}=0.11)$ and by $(\mathrm{B})$ interval time between prior therapy and retreatment (days) $(\mathrm{r}=0.08, \mathrm{P}=0.79)$.

the most pronounced reduction in size. A 64-year-old man, who was one of the 14 patients of the study cohort, was diagnosed with Ras wild-type rectal cancer with multiple liver and right pleural metastases. He first underwent resection of the primary lesion and received 10 courses of FOLFOX as a first-line therapy, and 24 courses of FOLFIRI and bevacizumab as a second-line therapy. Following disease progression subsequent to these two chemotherapies, the patient was treated with cetuximab and irinotecan as a third-line therapy. The metastatic tumors responded to this first cetuximab-based therapy and reduced by $60 \%$ in size (Fig. 3). Following 10 months of cetuximab-based therapy, contrast-enhanced computed tomography (CE-CT) revealed that the liver metastasis had enlarged. Then, the patient consecutively received regorafenib as a fourth-line therapy for 8 months and TAS102 as a fifth-line therapy for 4 months, and the tumor gradually progressed during the treatments. Considering the tumor progression, cetuximab and irinotecan was used as a sixth-line therapy. A total of 2 months later, CE-CT revealed that the liver metastasis had reduced by $60 \%$ in size again (Fig. 3). 
Table V. Cetuximab retreatment studies.

Study name

Study characteristics

JACCRO CC-08 (12) Santini et al (11)

CRICKET (10)

Present study

\begin{tabular}{lcccc}
\hline $\mathrm{n}$ & 34 & 39 & 26 & 14 \\
Median number of therapeutic lines (range) & 3 & $4(3-7)$ & 3 & $5(3-6)$ \\
Adjunct chemotherapy regimen & CPT11 & CPT11/FOLFIRI & CPT11 & CPT11 \\
$\begin{array}{l}\text { Median interval time between initial and } \\
\text { cetuximab retreatment, months (range) }\end{array}$ & - & $6(2-12)$ & $13(6-37)$ \\
Median PFS, months & 2.4 & 6.6 & - & 4.2 \\
Objective response rate (\%) & $1(3)$ & $21(54)$ & $6(23)$ & $3(21)$ \\
Disease control rate (\%) & $19(56)$ & $35(90)$ & $14(54)$ & $10(71)$ \\
\hline
\end{tabular}

PFS progression-free survival; CPT-11, irinotecan; FOLFIRI, folinic acid, fluorouracil and irinotecan.
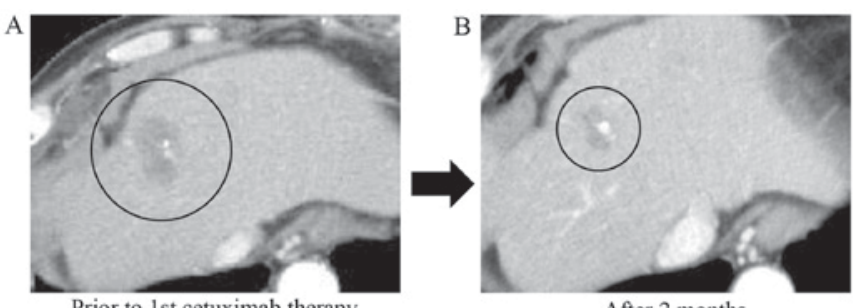

Prior to 1st cetuximab therapy
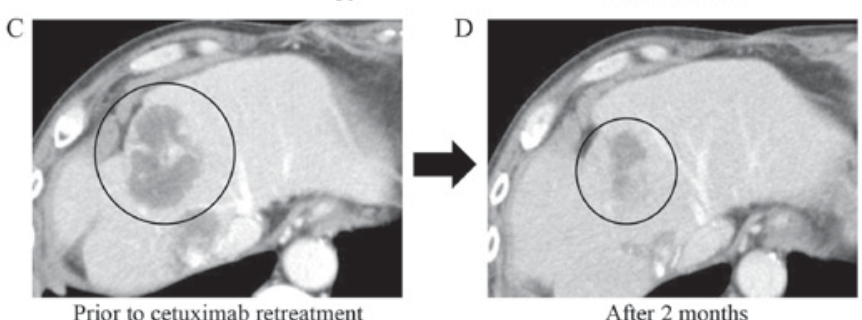

Figure 3. Clinical course of a metastatic liver tumor. Contrast enhanced computed tomography images demonstrating partial response of colorectal cancer liver metastasis to cetuximab and irinotecan treatment. (A) Prior to first cetuximab therapy (third-line therapy). (B) A total of 2 months following initial cetuximab therapy. (C) Prior to cetuximab retreatment (sixth-line therapy). (D) A total of 2 months following cetuximab retreatment. Black circles indicate location of the tumor.

\section{Discussion}

In the present study, it was identified that cetuximab retreatment was as effective and tolerable therapy in patients with mCRC who had previously exhibited disease progression following an initial clinical benefit to first-line cetuximab-based therapy. A small number of previous reports have demonstrated the clinical benefit of anti-EGFR retreatment in patients with $\mathrm{mCRC}$ (9-11). Table V summarizes the results of 3 previous phase II studies of cetuximab retreatment in patients with $\mathrm{mCRC}$. Patients with $\mathrm{mCRC}$ who received initial first-line cetuximab-based therapy and exhibited stable disease for at least 6 months or clinical response were included in the 3 studies (UMIN000010638 and NCT02296203) (10-12). In these studies, cetuximab retreatments involved combinations with irinotecan monotherapy or FOLFIRI. In the present study, irinotecan was administered with weekly cetuximab retreatment to all patients, and 3 of the patients received an irinotecan-based regimen immediately prior to the retreatment. The effectiveness of the combination of cetuximab and irinotecan in patients with $\mathrm{mCRC}$ with tumors refractory to irinotecan was indicated in a previous randomized trial that suggested that cetuximab may circumvent irinotecan resistance (17). Previous retreatment studies revealed an ORR of $3-54 \%$, DCR of $54-90 \%$ and median PFS of 2.4-6.6 months (10-12). The efficacy in the present study was equivalent to that in these previous studies, with an ORR of $21 \%$, DCR of $71 \%$ and median PFS of 4.4 months, even though the present study included 2 patients who received prior cetuximab as third-line therapy. Practically, the previous studies have demonstrated the efficacy of retreatment with anti-EGFR antibodies in the salvage-line treatment of mCRC; however, there is no defined rationale that re-challenge therapy affects tumors that have already been demonstrated as resistant to the same therapy.

Previously, mutations in the KRAS gene have been detected in circulating tumor DNA in patients with KRAS wild-type mCRC who exhibited disease progression during anti-EGFR therapy (18). Mathematical modeling indicated that the mutations were present in expanded subclones prior to the initiation of anti-EGFR therapy (18). These data support the theory that the mechanism of resistance to anti-EGFR therapy may arise from heterogeneity among CRC tumors. On the basis of this hypothesis, administering a different treatment that is not affected by KRAS status subsequent to the development of acquired anti-EGFR resistance may allow the dominant $K R A S$ wild-type clone to repopulate and regain sensitivity to anti-EGFR therapy.

A retrospective study by Liu et al (13) investigated the efficacy of an anti-EGFR-containing regimen in phase I/II clinical trials in patients with $K R A S$ wild-type mCRC exhibiting disease progression following initial cetuximab or panitumumab treatment (12). Patients who responded to prior cetuximab or panitumumab regimens were more likely to exhibit a clinical benefit to the retreatment compared with the non-responders. Additionally, prior responders with longer interval lengths between initial anti-EGFR therapy and anti-EGFR retreatment were 
more likely to respond positively to anti-EGFR retreatment compared with a shorter time interval. Recently, a number of limited studies demonstrated that the preceding treatment of anti-EGFR treatment may influence the efficacy of anti-EGFR treatment $(19,20)$. However, these studies did not provide a clear answer regarding the effect of the variation in interim treatments between initial anti-EGFR therapy and anti-EGFR retreatment. Unfortunately the sample size of the present study was too small to investigate this point further.

Conversely, Wadlow et al (21) prospectively treated 20 patients with KRAS wild-type mCRC by administering panitumumab followed by cetuximab subsequent to disease progression, and demonstrated that no patients responded and $45 \%$ exhibited the best response of SD (21). The present study did not demonstrate any benefit of administering panitumumab following disease progression subsequent to initial cetuximab treatment, and there was no interval therapy between cetuximab and panitumumab treatment. In the present study, the median interval time between the last cycle of initial cetuximab-based therapy and the first cycle of cetuximab retreatment was 12.6 months. Therefore, the fact that the ORR was $21.4 \%$ and the PFS was 4.4 months without any severe adverse events may be considered a sufficient response. It was also demonstrated that there was a more marked correlation between the duration of the initial cetuximab therapy and the efficacy of retreatment compared with the interval length between initial therapy and retreatment ( $\mathrm{r}=0.45$ vs. 0.11 ).

The data of the present study suggests that, to obtain any clinical benefit from anti-EGFR retreatment in patients with mCRC who exhibited disease progression following initial anti-EGFR-based therapy, it is important to select patients who demonstrate a good response to initial anti-EGFR-based therapy and/or those who have longer interval lengths between initial therapy and retreatment. To evaluate additional predictive factors of the response to anti-EGFR retreatment, additional clinical trials are required to examine the changes in biomarker expression of RAS mutations following initial anti-EGFR therapy using liquid biopsy, and to analyze its effect on the refractory tumor.

To conclude, cetuximab retreatment in patients with KRAS wild-type $\mathrm{mCRC}$ who exhibited disease progression following initial cetuximab-based therapy may provide greater clinical benefit to patients who were good responders with longer treatment durations in initial cetuximab-based therapy.

\section{Acknowledgements}

The authors would like to thank Ms. Hiroko Hamada and Ms. Midori Hashimoto (Okayama Rosai Hospital, Okayama, Japan) for their secretarial assistance.

\section{Funding}

No funding was received.

\section{Availability of data and materials}

The analyzed datasets generated during the study are available from the corresponding author upon reasonable request.

\section{Authors' contributions}

HT, YM and TN conceived and designed the study. HT wrote the paper. MA, RY, NW, FU, MI and KY analyzed the data and critically revised the manuscript. All authors were involved in data interpretation and writing the manuscript and have all read and approved the final version of the manuscript.

\section{Ethics approval and consent to participate}

The Institutional Review Board of Okayama Rosai Hospital approved the present study. Written informed consent was obtained from all patients who participated.

\section{Patient consent for publication}

Not applicable.

\section{Competing interests}

The authors declare that they have competing interests.

\section{References}

1. Matsuda A, Matsuda T, Shibata A, Katanoda K, Sobue T and Nishimoto H; Japan Cancer Surveillance Research Group: Cancer incidence and incidence rates in Japan in 2008: A study of 25 population-based cancer registries for the monitoring of cancer incidence in Japan (MCIJ) project. Jpn J Clin Oncol 44: 388-396, 2014.

2. Watanabe T, Itabashi M, Shimada Y, Tanaka S, Ito Y, Ajioka Y, Hamaguchi T, Hyodo I, Igarashi M, Ishida H, et al: Japanese Society for Cancer of the Colon and Rectum (JSCCR) guidelines 2014 for treatment of colorectal cancer. Int J Clin Oncol 20: 207-239, 2015.

3. National Comprehensive Cancer Network (NCCN): NCCN Guidelines for Colon cancer (version 2, 2017). http://www.ncen. org/professionals/physician_gls/f_guidelines.asp. Accessed November 09, 2017.

4. Van Cutsem E, Cervantes A, Adam R, Sobrero A, Van Krieken JH, Aderka D, Aranda Aguilar E, Bardelli A, Benson A, Bodoky G, et al: ESMO consensus guidelines for the management of patients with metastatic colorectal cancer. Ann Oncol 27: 1386-1422, 2016.

5. Douillard JY, Oliner KS, Siena S, Tabernero J, Burkes R, Barugel M, Humblet Y, Bodoky G, Cunningham D, Jassem J, et al: Panitumumab-FOLFOX4 treatment and RAS mutations in colorectal cancer. N Engl J Med 369: 1023-1034, 2013.

6. Stintzing S, Modest DP, Rossius L, Lerch MM, von Weikersthal LF, Decker T, Kiani A, Vehling-Kaiser U, Al-Batran SE, Heintges T, et al: FOLFIRI plus cetuximab versus FOLFIRI plus bevacizumab for metastatic colorectal cancer (FIRE-3): A post-hoc analysis of tumour dynamics in the final RAS wild-type subgroup of this randomised open-label phase 3 trial. Lancet. Oncol 17: 1426-1434, 2016.

7. Mayer RJ, Van CutsemE,FalconeA, YoshinoT, Garcia-CarboneroR, Mizunuma N, Yamazaki K, Shimada Y, Tabernero J, Komatsu Y, et al: Randomized trial of TAS-102 for refractory metastatic colorectal cancer. N Engl J Med 372: 1909-1919, 2015.

8. Grothey A, Van Cutsem E, Sobrero A, Siena S, Falcone A, Ychou M, Humblet Y, Bouché O, Mineur L, Barone C, et al: Regorafenib monotherapy for previously treated metastatic colorectal cancer (CORRECT): An international, multicentre, randomised, placebo-controlled, phase 3 trial. Lancet 381: 303-312, 2013.

9. Takeuchi N, Koike K, Yoshida S, Kudo A, Sekiguchi N, Nakayama A, Kubota K, Rokuhara T and Kitahara M: Treatment of metastatic refractory colorectal cancer following regorafenib failure. Mol Clin Oncol 7: 308-312, 2017.

10. Rossini D, Santini D, Cremolini C, Salvatore L, Lonardi S, Aquila ED, Aprile G, Loupakis F, Vincenzi B, Battaglin F, et al: Rechallenge with cetuximab + irinotecan in 3rd-line in RAS and BRAF wild-type metastatic colorectal cancer (mCRC) patients with acquired resistance to 1st-line cetuximab + irinotecan: The phase II CRICKET study by GONO. Ann Oncol 28 (Suppl3): iii1-iii12, 2017. 
11. Santini D, Vincenzi B, Addeo R, Garufi C, Masi G, Scartozzi M, Mancuso A, Frezza AM, Venditti O, Imperatori M, et al: Cetuximab rechallenge in metastatic colorectal cancer patients: How to come away from acquired resistance? Ann Oncol 23: 2313-2318, 2012.

12. Tsuji A, Eto T, Masuishi T, Satake HS, Segawa Y, Tanioka H, Hara H, Kotaka M, Sagawa T. Watanabe T, et al: Phase II study of third-line cetuximab rechallenge in patients with metastatic wild-type K-RAS colorectal cancer who achieved a clinical benefit in response to first-line cetuximab plus chemotherapy (JACCRO CC-08). Ann Oncol 27: 510P, 2016.

13. Liu X, George GC, Tsimberidou AM, Naing A, Wheler JJ, Kopetz S, Fu S, Piha-Paul SA, Eng C, Falchook GS, et al: Retreatment with anti-EGFR based therapies in metastatic colorectal cancer: Impact of intervening time interval and prior anti-EGFR response. BMC Cancer 15: 713, 2015.

14. National Cancer Institute: Common Terminology Criteria for Adverse Events (CTCAE) v4.0. http://ctep.cancer.gov/protocolDevelopment/electronic_applications/ctc.htm\#ctc_40. Accessed May 17, 2010.

15. Eisenhauer EA, Therasse P, Bogaerts J, Schwartz LH, Sargent D, Ford R, Dancey J, Arbuck S, Gwyther S, Mooney M, et al: New response evaluation criteria in solid tumours: Revised RECIST guideline (version 1.1). Eur J Cancer 45: 228-247, 2009.

16. Kanda Y: Investigation of the freely available easy-to-use software 'EZR' for medical statistics. Bone Marrow Transplant 48: 452-458, 2013

17. Cunningham D, Humblet Y, Siena S, Khayat D, Bleiberg H, Santoro A, Bets D, Mueser M, Harstrick A, Verslype C, et al: Cetuximab monotherapy and cetuximab plus irinotecan in irinotecan-refractory metastatic colorectal cancer. N Engl J Med 351: $337-345,2004$
18. Diaz LA Jr, Williams RT, Wu J, Kinde I, Hecht JR, Berlin J, Allen B, Bozic I, Reiter JG, Nowak MA, et al: The molecular evolution of acquired resistance to targeted EGFR blockade in colorectal cancers. Nature 486: 537-540, 2012.

19. Price T, Kim TW, Li J, Cascinu S, Ruff P, Suresh AS, Thomas A, Tjulandin S, Guan X and Peeters M: Final results and outcomes by prior bevacizumab exposure, skin toxicity, and hypomagnesaemia from ASPECCT: Randomized phase 3 non-inferiority study of panitumumab versus cetuximab in chemorefractory wild-type KRAS exon 2 metastatic colorectal cancer. Eur J Cancer 68: 51-59, 2016.

20. Taniguchi H, Komori A, Narita Y, Kadowaki S, Ura T, Andoh M, Yatabe Y, Komori K, Kimura K, Kinoshita T and Muro K: A short interval between bevacizumab and anti-epithelial growth factor receptor therapy interferes with efficacy of subsequent anti-EGFR therapy for refractory colorectal cancer. Jpn J Clin Oncol 46: 228-233, 2016.

21. Wadlow RC, Hezel AF, Abrams TA, Blaszkowsky LS, Fuchs CS, Kulke MH, Kwak EL, Meyerhardt JA, Ryan DP, Szymonifka J, et al: Panitumumab in patients with KRAS wild-type colorectal cancer after progression on cetuximab. Oncologist 17: 14, 2012

This work is licensed under a Creative Commons Attribution-NonCommercial-NoDerivatives 4.0 International (CC BY-NC-ND 4.0) License. 